\title{
The effect of an emollient with benfothiamine and Biolin prebiotic on the improvement of epidermal skin function
}

\author{
Joanna Narbutt, Igor A. Bednarski, Aleksandra Lesiak \\ Dermoklinika Medical Centre, Lodz, Poland
}

Adv Dermatol Allergol 2016; XXXIII (3): 224-231

DOI: $10.5114 / a d a .2016 .60616$

\begin{abstract}
Introduction: Complications of diabetes can damage internal organs and the skin. Diabetic skin, irritated and dry, is susceptible to skin infections. However little is known about influence of emollients on biophysical changes in skin during diabetes.

Aim: To evaluate clinical skin changes after application of emollients with benfothiamine and Biolin prebiotic and to assess changes in biophysical parameters of the skin before and 4 weeks after daily application of an emollient. Material and methods: We recruited 50 patients with diabetes mellitus type 1 (DM1) or type 2 (DM2). All participants applied emollients on their left forearms and left shins for 4 weeks. The biophysical properties: $\mathrm{pH}$, transepidermal water loss (TEWL), hydration of the stratum corneum and sebum content were measured and compared to those before enrollment to the study, after $1 \mathrm{~h}, 1$ week and 4 weeks after application of an emollient.

Results: After 4 weeks of treatment, there was an increase in skin hydration ( $40.61 \pm 19.03$ vs. $48.83 \pm 15.51), \mathrm{pH}(5.11$ \pm 0.56 to $5.27 \pm 0.48)$ and sebum content $(22.16 \pm 8.67$ to $63.99 \pm 25.41)$ and a decrease in TEWL (12.54 \pm 5.6 vs. 9.85 $\pm 5.69 \mathrm{~g} / \mathrm{m}^{2} / \mathrm{h}$ ) on forearms ( $p<0.05$ for all comparisons). On lower legs, significant changes in skin hydration (37.21 \pm 14.01 vs. $43.95 \pm 12.67)$, pH ( $5.04 \pm 0.57$ to $5.31 \pm 0.49)$, sebum content ( $25.82 \pm 10.46$ to $72.63 \pm 31.23)$ and TEWL (8.87 \pm 4.05 vs. $7.39 \pm 3.22 \mathrm{~g} / \mathrm{m}^{2} / \mathrm{h}$ ) were observed ( $p<0.05$ for all comparisons).

Conclusions: Our study provides an insight into changes in diabetic skin after application of an emollient. To our knowledge, this is the first report on the emollient containing benfothiamine and Biolin prebiotic and its influence on biophysical parameters of epidermis.
\end{abstract}

Key words: diabetic skin, emollient, skin dryness.

\section{Introduction}

Diabetes mellitus (DM) is a group of chronic and systemic diseases affecting many internal organs. A key finding in diabetes is constant elevation of blood glucose (hyperglycemia) which may occur due to damage in Langerhans islet cells in the pancreas, resistance to insulin, and inappropriate hormone secretion. Genetic factors are also implicated in the development of diabetes. It is estimated that diabetes can affect about $8.3 \%$ of the general population [1] thus being a serious problem for healthcare systems throughout the world. Chronic hyperglycemia is affecting mostly blood vessels, kidneys, peripheral nerves and skin [2]. Skin complications are present in more than $1 / 3$ patients with diabe- tes. The direct cause of skin complications includes vasculopathy, accumulation of advanced glycation-end products and infection [2]. Localized itch, skin dryness and frequent bacterial and fungal infections are common problems in patients with diabetes. It is suspected that these problems are caused by changes in biophysical parameters of the skin including transepidermal water loss (TEWL), sebum and hydration of the stratum corneum. In diabetes there is an induction of advanced glycation-end products (AGEs) in the collagen of the dermis. Collagen glycation impairs (stiffens) its structure and function thus contributing to accelerated skin ageing [3, 4]. Established AGEs attach to specific receptors (receptor for AGE - RAGE). Intercommunication be-

Address for correspondence: Joanna Narbutt MD, PhD, Dermoklinika Medical Centre, 93 Kosciuszki St, 90-436 Lodz, Poland, phone: +48 692065 698, e-mail: joanna.narbutt@onet.pl Received: 15.04.2016, accepted: 27.05.2016. 
tween AGE and RAGE activates NADPH oxidase, one of the enzymes responsible for reactive oxygen species production (ROS), leading to oxidative stress, thus inducing proliferative response in endothelial cells as well as inflammatory and thrombotic reactions which can promote development of vasculopathy [5-7].

Another important and common, but underestimated problem in dermatological care in diabetes, is dry skin. The main cause of skin dryness is disruption in the epidermal barrier structure [8]. Functioning of the epidermal barrier relies mainly on stratum corneum (SC). Stratum corneum prevents excessive water loss and penetration of pathogens and allergens. However, research shows that diabetes influences epidermal barrier quality, which leads to, for example, a greater susceptibility to skin bacterial infections. A higher prevalence of skin and soft tissue infections (SSTI) caused by Staphylococcus aureus (SA) and Fournier's gangrene is well proven in diabetes [9]. Staphylococcus aureus is responsible for more SSTI cases than any other pathogen. In 2004, SA was responsible for $1 / 2$ of SSTI in North America, and $1 / 3$ of SSTI in Latin America and Europe [10]. It is suspected that a higher prevalence of SSTI in patients with diabetes is caused by mechanical dysfunction of the epidermal barrier [11], excessive skin colonization by bacteria [12] and altered humoral and cellular immune response [13]. However, it is impossible to describe all factors involved in skin susceptibility to infections in diabetes as this topic has not been fully evaluated yet.

There are no emollients developed for diabetic patients, and no research about the potential role of emollients in skin dryness prophylaxis in diabetes. Also, the problem of dry skin in diabetes is often neglected by general practitioners. Emollient addition to standard diabetes therapy may lower skin complications associated with elevated blood sugar [14]. According to the American Diabetic Association, use of emollients is highly recommended in diabetic skin care [15]. Employment of emollients can restore physiological lipid levels in the skin and reduce desquamation and pruritus $[16,17]$.

The primary aim of the study was to assess a statistically significant clinical improvement in forearms and lower legs skin condition after application of an emollient with benfothiamine and Biolin prebiotic (Emolium Diabetix) according to the physician's assessment. The secondary aim was to assess the improvement in biophysical parameters of skin including $\mathrm{pH}$, TEWL, hydration of the stratum corneum and sebum content in diabetic patients before and 4 weeks after daily application of an emollient. We also aimed to evaluate cosmetic properties, tolerance and satisfaction of the used product.

\section{Aim}

Thus, the aim of the study was to investigate clinical efficacy of Emolium Diabetix emollients in diabetic skin care.

\section{Material and methods}

The study was conducted in 50 patients with diabetes, $48 \%$ of them were males $(n=24)$ and $52 \%$ were females $(n=26)$. The mean age of patients was 56.2 \pm 9.26 years and the mean duration of diabetes (based on diagnosis) was $10.26 \pm 8.22$. Eighteen percent $(n=9)$ of patients suffered from diabetes type I and $82 \%(n=$ 41) from diabetes type II. All patients were enrolled into the study after meeting the following inclusion criteria: age between 18 and 65 years, diabetes for at least 1 year, having a healthy skin. Pregnant women, participants in other medical experiments, active smokers, people with peripheral oedema and neuropathy were excluded from the study. Treatment regimen involved daily application (2-times-a-day with $12 \mathrm{~h}$ between subsequent applications) of an emollient for 28 days. The biophysical measurements were made in strictly determined time points: before the first application, $1 \mathrm{~h}, 1$ week and 4 weeks after the first application. The study was approved by the local Ethics Committee (no. RNN/163/15/KE). All patients signed written consent before enrollment to the study.

Visual skin properties, both on the forearm and lower leg, were assessed using graduation tables (1-4 scale, 1 - lack of any changes, 2 - low severity changes, 3 moderate severity changes 4 - the greatest severity changes) in which skin irritation, erythema, desquamation, roughness and dryness were evaluated. Assessment of visual skin properties was made before enrollment to the study (t0), 1 week after the first application (t2) and 4 weeks after the first application (t4).

The biophysical parameters of the skin were measured at two different locations of the body: extensor surface of the left forearm and extensor surface of the left lower leg using non-invasive biophysical methods. Before taking the measurement, all participants were acclimatized for $20 \mathrm{~min}$. To estimate exact changes in the skin during the study we used Multi-Probe Adapter (Courage \& Khazaka Electronic, Cologne, Germany) with attached specific probes. Stratum corneum hydration was measured with a Corneometer ${ }^{\oplus}$ CM825 (Courage \& Khazaka Electronic, Cologne, Germany), skin pH - using Skin-pH-meter ${ }^{\oplus} \mathrm{PH} 905$ (Courage \& Khazaka Electronic, Cologne, Germany), the sebum content was evaluated by Sebumeter ${ }^{\circledast}$ SM 815 (Courage \& Khazaka Electronic, Cologne, Germany) by measuring the amount of fat on the surface of skin. Transepidermal water loss, a measure of the water barrier function was assessed using a Tewameter ${ }^{\circledast}$ TM300 (Courage \& Khazaka Electronic, Cologne, Germany).

After 4 weeks of treatment, patients' and doctors' satisfaction from the used product was also evaluated (on a 1-5 scale, where 1 - the lowest satisfaction, 5 - the highest satisfaction).

Additionally in 10 patients ( 5 M, 5 F, mean age 59.9 \pm 1.4 years, min 36 years, max. 64.1 years) measurement 
of skin autofluorescence (SAF) was performed with the use of AGE Reader (Diagnoptics BV, Groningen, The Netherlands). The test was done in the dark room, in room temperature on the inner part of the right forearm. In each patient, measurements were performed 3 times at two time points: before Emolium Diabetix application and 6 weeks after regular (2 daily) use of the investigated product.

\section{Statistical analysis}

Data were analyzed using Statsoft Statistica 12 software. To evaluate differences between groups we used t-Student, Kruskall-Wallis, Wilcoxon singed rank tests and Friedman ANOVA. Based on the number of patients included into the study, the Shapiro-Wilk test was used to evaluate distribution. Values of $p<0.05$ were considered as statistically significant.
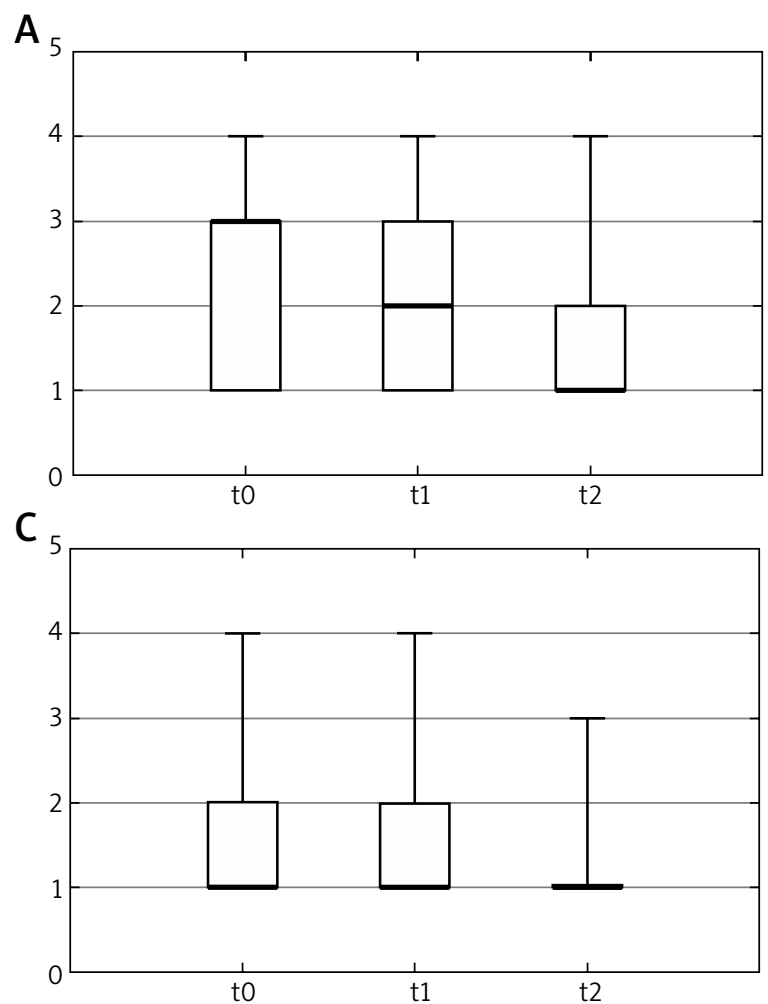

E

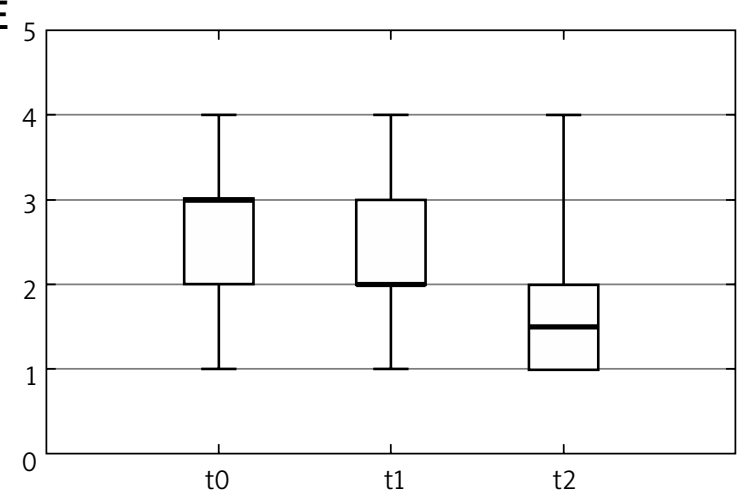

\section{Results}

There were no significant differences in response to treatment, according to gender of the subject. Also, the type of diabetes does not seem to influence response to treatment $(p>0.05)$.

Dermatological examination of the forearms and lower legs with graduation tables has shown that statistically significant changes $(p<0.05)$ occurred both after 1 and after 4 weeks of application of the emollient. After 4 weeks of the emollient application all visual parameters: irritation, erythema, desquamation, roughness and dryness decreased significantly $(p<0.05)$. Detailed results for each body site are shown in Figures 1 and 2.

After 4 weeks of treatment, we observed a significant $(p<0.05)$ increase in skin hydration both on forearms (40.61 \pm 19.03 vs. $48.83 \pm 15.51, p<0.05)$ and lower
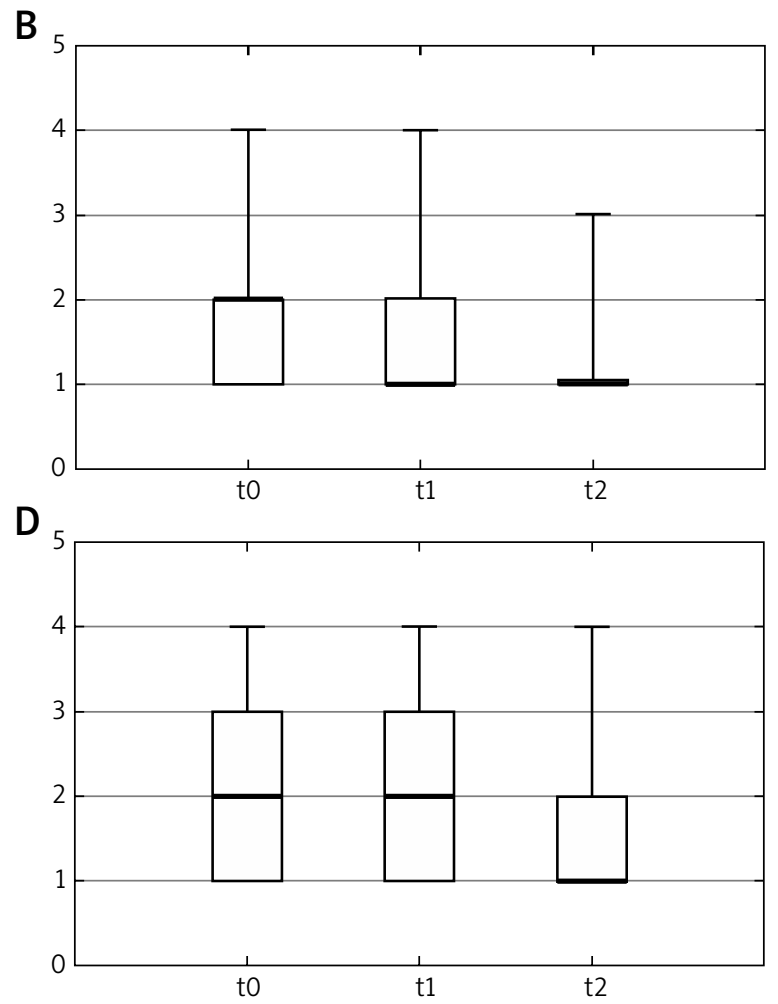

Figure 1. Change in irritation (A), erythema (B), desquamation $(C)$, roughness $(D)$ and dryness $(E)$ on the forearms during the study. Data presented as medians (thick black lines), interquartile ranges (boxes). Minimal and maximal values are indicated as error bars. Measurements were made before enrollment to the study (t0), after 1 week of treatment (t1) and after 4 weeks of treatment (t2). Significance of differences estimated with Wilcoxon's signed rank test 
A

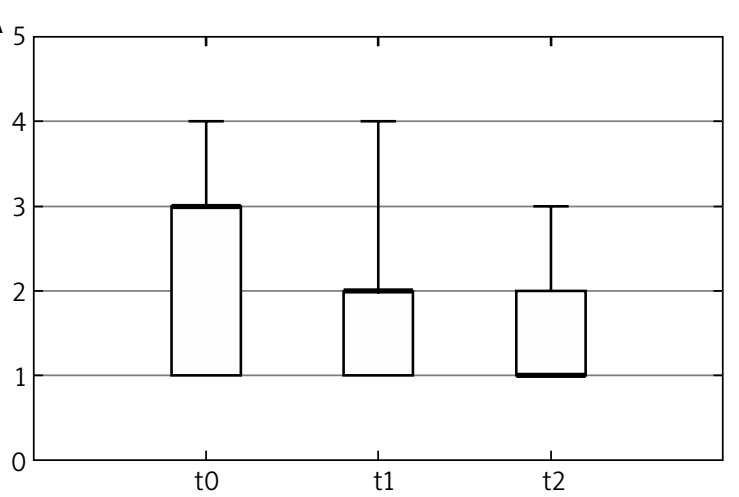

C

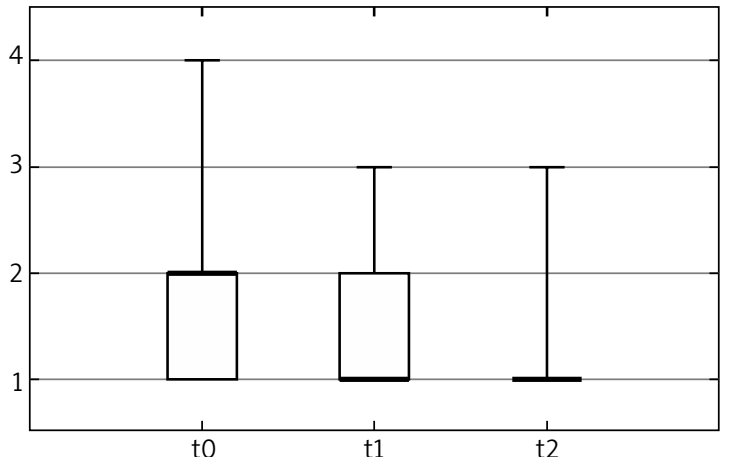

E

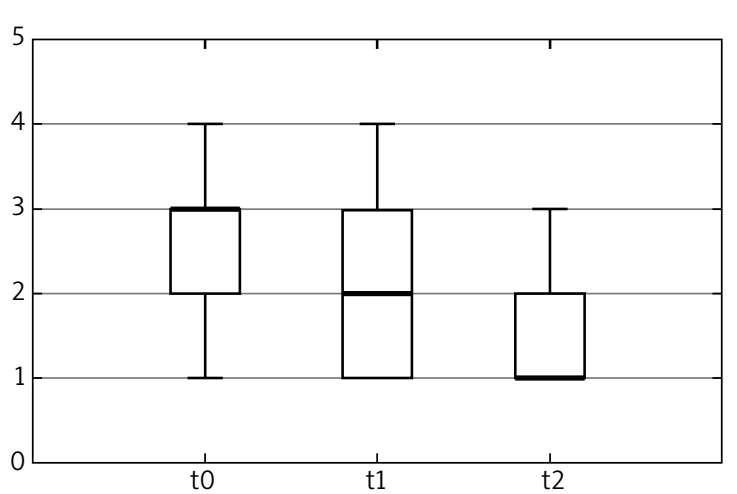

legs $(37.21 \pm 14.01$ vs. $43.95 \pm 12.67, p<0.05)$. Skin $\mathrm{pH}$ increased significantly on forearms $(5.11 \pm 0.56$ to 5.27 $\pm 0.46, p<0.05)$ and lower legs $(5.04 \pm 0.57$ to $5.31 \pm 0.49$, $p<0.05)$. The skin sebum level increased from 22.16 \pm 8.67 to $63.99 \pm 25.41(p<0.05)$ on forearms and from $25.82 \pm 10.46$ to $72.63 \pm 31.23(p<0.05)$ on lower legs. We also observed a decrease in TEWL on forearms (12.54 \pm 5.6 vs. $9.85 \pm 5.69, p<0.05)$, and on the lower legs (8.87 \pm 4.05 vs. $\left.7.39 \pm 3.22 \mathrm{~g} / \mathrm{m}^{2} / \mathrm{h}, p<0.05\right)$.

All the measurements are shown in Figures 3 and 4 .

Skin autofluorescence results are shown in Table 1.

Mean values of the $1^{\text {st }}$ and $2^{\text {nd }}$ measurements were $3.34 \pm 0.42$ vs. $2.16 \pm 0.48$, respectively, however there were no significant differences between both measurements $(p=0.42)$.
$B_{5}$

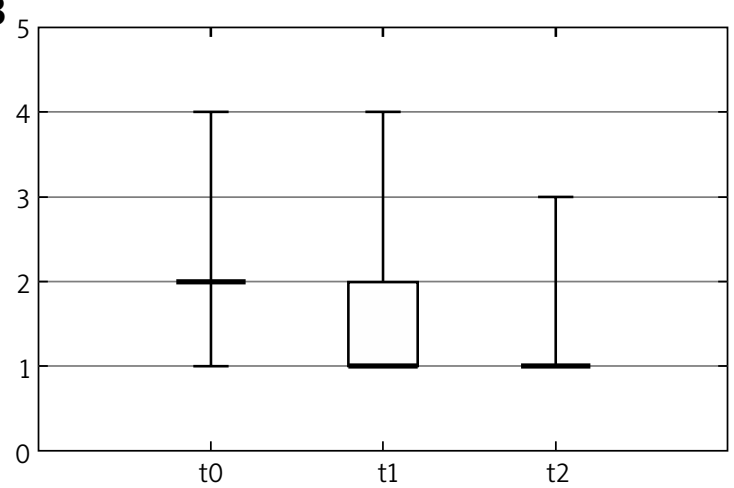

$D_{5}$

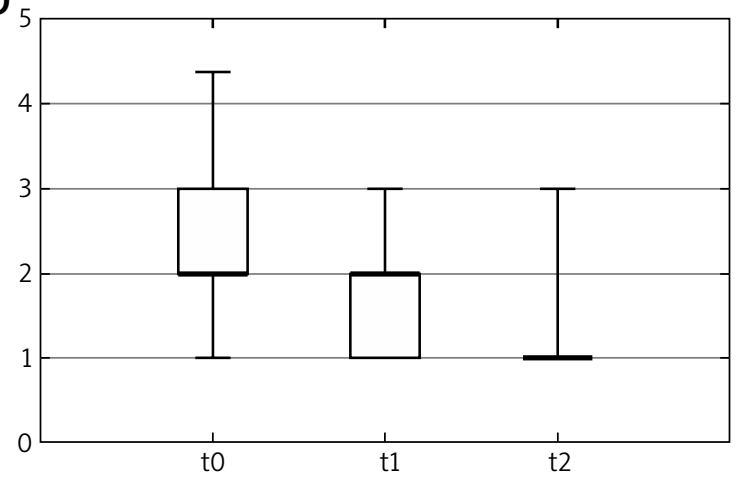

Figure 2. Change in irritation (A), erythema (B), desquamation $(C)$, roughness $(D)$ and dryness $(E)$ on the lower legs during the study. Data presented as medians (thick black lines), interquartile ranges (boxes). Minimal and maximal values are indicated as error bars. Measurements were made before enrollment to the study (t0), after 1 week of treatment (t1) and after 4 weeks of treatment (t2). Significance of differences estimated with Wilcoxon's signed rank test

Table 1. Skin autofluorescence results

\begin{tabular}{lcc}
\hline Value & First measurement & $\begin{array}{c}\text { Secound } \\
\text { measurement }\end{array}$ \\
\hline Minimum & 1.500 & 1.900 \\
\hline $25 \%$ percentile & 1.775 & 2.050 \\
\hline Median & 2.150 & 2.100 \\
\hline $75 \%$ percentile & 2.525 & 2.675 \\
\hline Maximum & 2.900 & 3.100 \\
\hline Mean & 2.160 & 2.340 \\
\hline Std. deviation & 0.4812 & 0.4274 \\
\hline Std. error & 0.1522 & 0.1352 \\
\hline
\end{tabular}



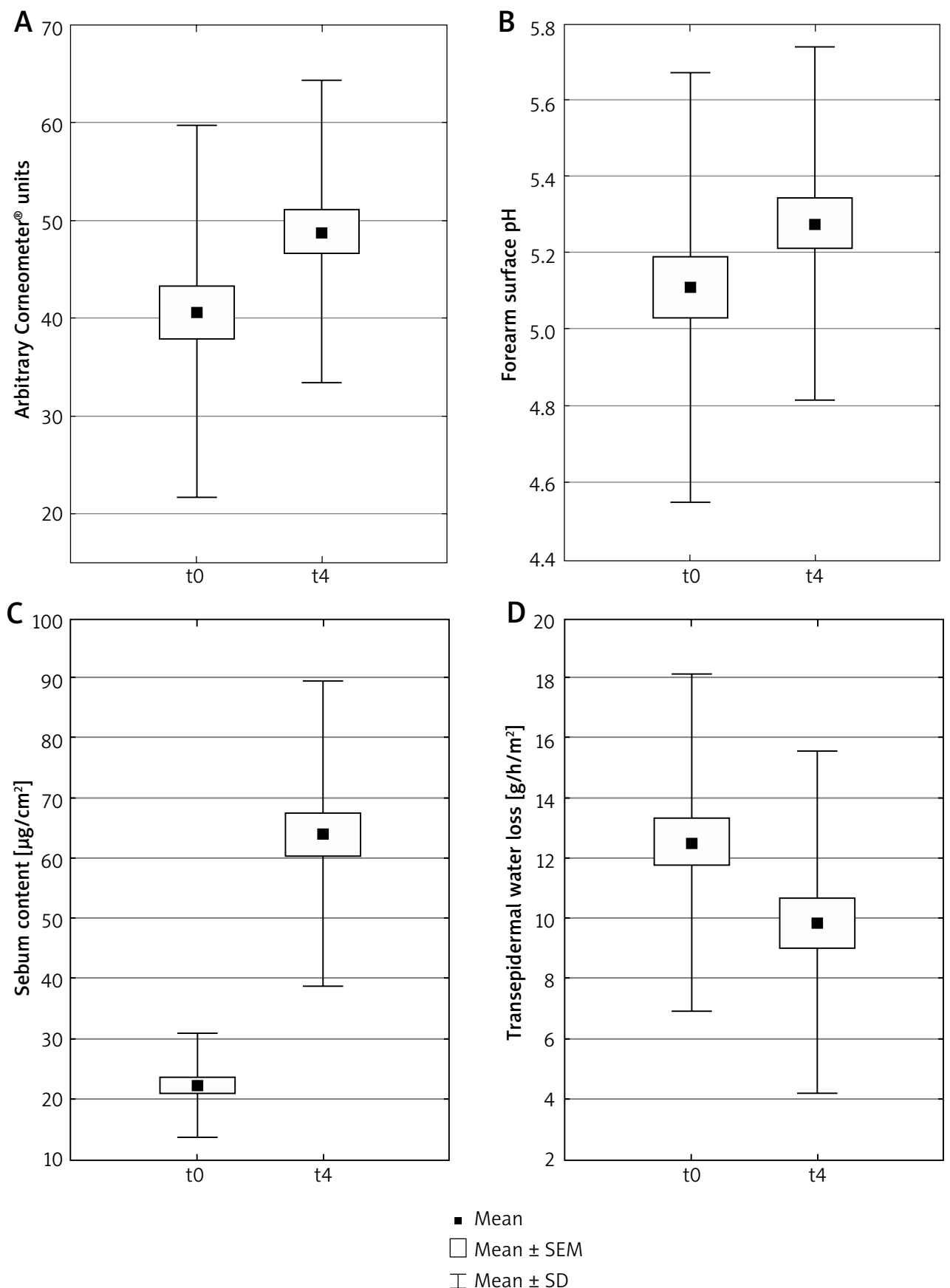

Figure 3. Change in skin hydration (A), pH (B), sebum content $(\mathbf{C})$ and transepidermal water loss (D) before (t0) and after 4 weeks of emollient application on forearms (t4). Data presented as means (small black squares), standard error of measurement indicated as black rectangles and standard deviation indicated as error bars. Significance of differences estimated with Wilcoxon's signed rank test

After 4 weeks, satisfaction of used products was evaluated. Ninety percent of patients rated Emolium Diabetix with a good or very good note (4 or 5). $10 \%$ of patients rated Emolium Diabetix as satisfactory (3). In $56 \%$ of cases the physician rated the summarized toler- ance of used products as very good (5) and in 44\% of cases as good (4).

The used products were well tolerated according to patients' and physicians' observations. During the study no undesirable effects occurred in any patient. 

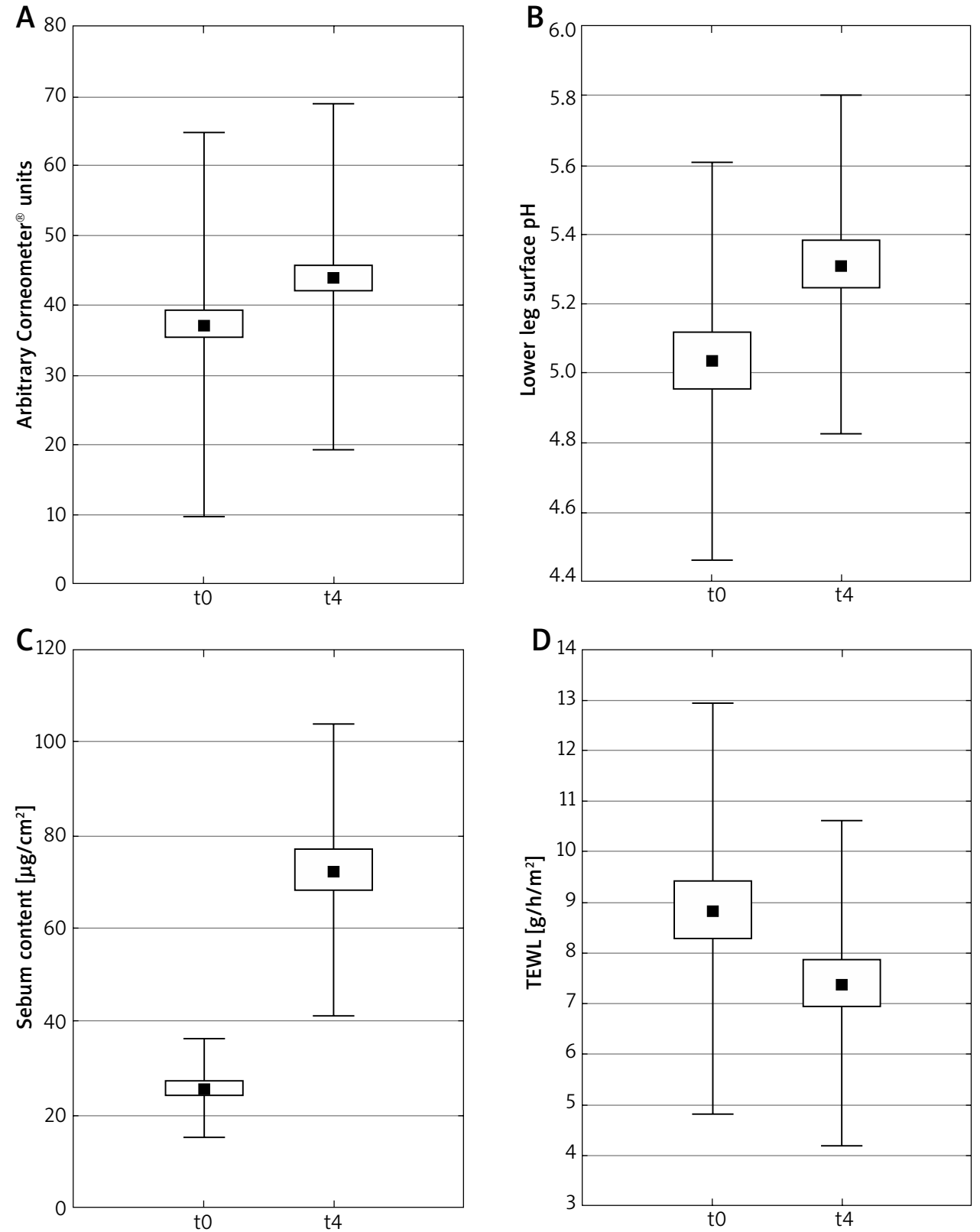
- Mean
$\square$ Mean \pm SEM
I Mean \pm SD

Figure 4. Change in skin hydration (A), pH (B), sebum content (C) and transepidermal water loss (D) before (t0) and after 4 weeks of emollient application on lower legs (t4). Data presented as means (small black squares), standard error of measurement indicated as black rectangles and standard deviation indicated as error bars. Significance of differences estimated with $t$-Student paired test and Wilcoxon's signed rank test

\section{Discussion}

Impairment of the epidermal barrier results in changes in skin biophysical parameters such as TEWL or sebum level. In spite of knowledge of the subject, there is very little research done on changes in skin biophysical parameters in diabetes and there are no literature data on the role of emollient therapy in diabetic skin care. Special care of diabetic skin seems to be extremely important as it is commonly known that hyperglycemia and insulin 
resistance may impair epidermis homeostasis by lowering skin hydration and dysregulation of sebaceous glands function.

Transepidermal water loss elevation may indicate potential epidermal barrier dysfunction [18], however investigations about its biological function in diabetic skin are controversial. Lack of deviation in TEWL levels does not exclude any damage to the epidermal barrier [19]. Moreover, the TEWL level depends on many factors, for example local climate, air humidity, season, age of the patient and regional blood flow [20-22]. It has not been proven yet that TEWL changes in diabetes [23, 24]. It is supposed that water loss from the epidermis is likely caused by hyperglycemia and blood hyperosmolarity, rather than water evaporation from the skin [23-25]. In our research, we describe a significant decline in TEWL after systematic application of an emollient, however only on the forearms. The result seems to be logical, nevertheless it is hard to determine its exact significance.

Sebaceous glands (SG) are also playing a role in development of dry skin. SG are producing sebum which consists of triacylglicerides, squalene and waxes. This hydrophobic layer created by sebum, helps protect skin surface against bacteria and fungi and prevents excessive water loss (evaporation) from the skin surface. Research conducted on diabetic patients and streptozocin-induced-diabetes mice showed decreased sebum production [24]. We found that the level of skin surface lipid levels increases during therapy. We suspect that usage of an emollient restores disturbed skin sebum levels, thus improving all other biophysical parameters. In other studies it was shown that emollients can improve stratum corneum hydration which is decreased in various skin pathologies, e.g. in sensitive and diabetic skin [26]. Disruption of the epidermal permeability barrier enables percutaneous penetration of a variety of substances including toxins, allergens and infection factors [27, 28] leading to irritation, infections and pruritus. In our study, regular use of Emolium Diabetix emollients statistically improved hydration of stratum corneum after 4 weeks of application. We may assume that such an emollient regimen would provide a clinical improvement of the skin condition and a reduction in skin dryness. Beneficial effects of the applied emollient on stratum corneum hydration provides another rationale for their usage in treating diabetic skin. During emollient use we observed normalization of skin $\mathrm{pH}$ which probably would result in an improved epidermal skin integrity and limitation of bacterial infections [29, 30].

\section{Conclusions}

Prophylaxis of skin dryness and pruritus is based on regular use of emollients as it leads to restoration of the epidermal barrier, reduces inflammation, skin infections and pruritus. Despite being widely used in dermatologi- cal practice, there are no literature data on its usefulness in diabetic skin care. To our knowledge, this is the first report specifically dedicated to diabetic skin emollients that contain benfothiamine and Biolin prebiotic, describing its influence on the biophysical parameters of epidermis. They showed a beneficial effect, however the final therapeutic success can be achieved only when they are used regularly.

\section{Conflict of interest}

The paper was supported by Sanofi Poland.

\section{References}

1. Shi Y, Hu FB. The global implications of diabetes and cancer. Lancet 2014; 383: 1947-8.

2. American Diabetes Association. Diagnosis and classification of diabetes mellitus. Diabetes Care 2013; 36: 67-74.

3. Yamagishi S, Matsui T. Advanced glycation end products, oxidative stress and diabetic nephropathy. Oxid Med Cell Longev 2010; 3: 101-8.

4. Fukami K, Yamagishi S, Okuda S. Role of AGEs-RAGE system in cardiovascular disease. Curr Pharm Des 2014; 20: 2395402.

5. Yamagishi S, Nakamura K, Matsui T, et al. Agents that block advanced glycation end product (AGE)-RAGE (receptor for AGEs)-oxidative stress system: a novel therapeutic strategy for diabetic vascular complications. Expert Opin Investig Drugs 2008; 17: 983-96.

6. Stern D, Yan SD, Yan SF, Schmidt AM. Receptor for advanced glycation endproducts: a multiligand receptor magnifying cell stress in diverse pathologic settings. Adv Drug Deliv Rev 2002; 54: 1615-25.

7. Bierhaus A, Hofmann MA, Ziegler R, Nawroth PP. AGEs and their interaction with AGE-receptors in vascular disease and diabetes mellitus. I. The AGE concept. Cardiovasc Res 1998; 37: 586-600.

8. Böhme M, Svensson A, Kull I, Wahlgren CF. Hanifin's and Rajka's minor criteria for atopic dermatitis: which do 2-yearolds exhibit? J Am Acad Dermatol 2000; 43: 785-9.

9. DiNubile MJ, Lipsky BA. Complicated infections of skin and skin structures: when the infection is more than skin deep. J Antimicrob Chemother 2004; 53: 37-50.

10. Moet GJ, Jones RN, Biedenbach DJ, et al. Contemporary causes of skin and soft tissue infections in North America, Latin America, and Europe: report from the SENTRY Antimicrobial Surveillance Program (1998-2004). Diagn Microbiol Infect Dis 2007; 1: 7-13.

11. Wanke I, Skabytska Y, Kraft B, et al. Staphylococcus aureus skin colonization is promoted by barrier disruption and leads to local inflammation. Exp Dermatol 2013; 22: 153-5.

12. Redel H, Gao Z, Aleksyenko AV, et al. Quantitation and composition of cutaneous microbiota in diabetic and non-diabetic men. J Infect Dis 2013; 207: 1105-14.

13. Gallacher SJ, Thomson G, Fraser WD, et al. Neutrophil bactericidal function in diabetes mellitus: evidence for association with blood glucose control. Diabet Med 1995; 10: 916-20.

14. Seité S, Khemis A, Rougier A, Ortonne J. Importance of treatment of skin xerosis in diabetes. J Eur Acad Dermatol Venereol 2011; 25: 607-9. 
15. Proksch E. The role of emollients in the management of diseases with chronic dry skin. Skin Pharmacol Physiol 2008; 21: 75-80.

16. Lodén M. Role of topical emollients and moisturizers in the treatment of dry skin barrier disorders. Am J Clin Dermatol 2003; 4: 771-88.

17. Lodén M. The clinical benefit of moisturizers. J Eur Acad Dermatol Venereol 2005; 19: 672-88.

18. Pinnagoda J, Tupkek RA, Agner T, Serup J. Guidelines for transepidermal water loss (TEWL) measurement. Contact Dermatitis 1990; 22: 164-78.

19. Proksch E, Brandner JM, Jensen JM. The skin: an indispensable barrier. Exp Dermatol 2008; 17: 1063-72.

20. Black D, Del Pozo A, Lagarde JM, Gall Y. Seasonal variability in the biophysical properties of stratum corneum from different anatomical sites. Skin Res Technol 2000; 6: 70-6.

21. Ngo BT, Hayes KD, DiMiao DJ, et al. Manifestations of cutaneous diabetic microangiopathy. Am J Clin Dermatol 2005; 6: 225-37.

22. Dinh TL, Veves A. A review of the mechanisms implicated in the pathogenesis of the diabetic foot. Int J Low Extrem Wounds 2005; 4: 154-9.

23. Seirafi H, Farsinejad K, Firooz A, et al. Biophysical characteristics of skin in diabetes: a controlled study. J Eur Acad Dermatol Venereol 2009; 23: 146-9.

24. Sakai S, Kikuchi K, Satoh J, et al. Functional properties of stratum corneum in patients with diabetes mellitus: similarities to senile xerosis. Br J Dermatol 2005; 153: 319-23.

25. Chilcott RP, Dalton CH, Emmanuel AJ, et al. Transepidermal water loss does not correlate with skin barrier function in vitro. J Invest Dermatol 2002; 118: 871-5.

26. Seidenari S, Francomano M, Mantovani L. Baseline biophysical parameters in subjects with sensitive skin. Contact Dermatitis 1998; 38: 311-5.

27. Tsai JC, Guy RH, Thornfeldt CR, et al. Metabolic approaches to enhance transdermal drug delivery. 1. Effect of lipid synthesis inhibitors. J Pharm Sci 1996; 85: 643-8.

28. Elias PM, Tsai J, Menon GK, et al. The potential of metabolic interventions to enhance transdermal drug delivery. I Investig Dermatol Symp Proc 2002; 7: 79-85.

29. Harder J, Schröder JM, Gläser R. The skin surface as antimicrobial barrier: present concepts and future outlooks. Exp Dermatol 2013; 22: 1-5.

30. Hon KL, Wang SS, Pong NH, Leung TF. The ideal moisturizer: a survey of parental expectations and practice in childhoodonset eczema. J Dermatol Treat 2013; 24: 7-12. 